\title{
Holographic Rényi Entropy at High Energy Density
}

\author{
Xi Dong ${ }^{*}$ \\ Department of Physics, University of California, Santa Barbara, California 93106, USA
}

(Received 17 November 2018; published 1 February 2019)

\begin{abstract}
We show that Rényi entropies of subregions can be used to distinguish when the entire system is in a microcanonical ensemble from when it is in a canonical ensemble, at least in theories holographically dual to gravity. Simple expressions are provided for these Rényi entropies in a particular thermodynamic limit with high energy density and fixed fractional size of the subregion. Holographically, the Rényi entropies are determined by the areas of cosmic branes inserted into the bulk spacetime. They differ between a microcanonical and a canonical ensemble because the two ensembles provide different boundary conditions for the gravitational theory under which cosmic branes lead to different backreacted geometries. This is in contrast to the von Neumann entropy which is more coarse-grained and does not differentiate microcanonical ensembles from canonical ensembles.
\end{abstract}

DOI: 10.1103/PhysRevLett.122.041602

Introduction.-In a chaotic quantum system, the eigenstate thermalization hypothesis [1-3] posits that a finitely excited energy eigenstate behaves thermally when restricted to a subsystem much smaller than the entire system. In other words, few-body operators cannot distinguish an energy eigenstate from a suitable thermal state.

This raises an important question: are there more global probes that can differentiate an energy eigenstate from a thermal state? In particular, it is well motivated to consider probes defined in a subsystem $A$ whose fractional size $f \equiv V_{A} / V$ is finite in the thermodynamic limit. As a first step in studying this question, it is useful to address a related question: what probes on a subsystem can be used to distinguish when the entire system is in a microcanonical ensemble at fixed total energy from when it is in a canonical ensemble at an appropriate temperature?

The main purpose of this Letter is to answer this second question in holographic theories by showing that the Rényi entropies [4]

$$
S_{n}^{A} \equiv \frac{1}{1-n} \ln \operatorname{tr} \rho_{A}^{n}
$$

of any subsystem $A$ of finite fractional size differ between a microcanonical and a canonical ensemble for the entire system as long as $n \neq 1$. This suggests that, even when restricted to a subsystem, Rényi entropies have much more fine-grained information about the entire state than the von

Published by the American Physical Society under the terms of the Creative Commons Attribution 4.0 International license. Further distribution of this work must maintain attribution to the author(s) and the published article's title, journal citation, and DOI. Funded by SCOAP ${ }^{3}$.
Neumann entropy. By a happy coincidence, Rényi entropies (for integer $n \geq 2$ ) are much easier to measure experimentally than the von Neumann entropy, as recently demonstrated using ultracold atoms [5]. Moreover, the results of this Letter are motivated by similar observations made recently in Ref. [6] (which is itself motivated by Refs. [7,8]) for individual energy eigenstates instead of microcanonical ensembles, using arguments based on ergodicity and eigenstate thermalization rather than holography.

Our main technical tool is a simple, geometric prescription derived in Refs. [9,10] for calculating Rényi entropies using gauge-gravity duality [11-13]. It says that in quantum field theories (QFTs) with a dual gravitational description in a bulk spacetime with one additional dimension, a refined version of the Rényi entropy introduced in Ref. [10],

$$
\tilde{S}_{n}^{A} \equiv n^{2} \partial_{n}\left(\frac{n-1}{n} S_{n}^{A}\right)=-n^{2} \partial_{n}\left(\frac{1}{n} \ln \operatorname{tr} \rho_{A}^{n}\right),
$$

is given by the area of a bulk codimension- 2 cosmic brane homologous to the subregion $A$ :

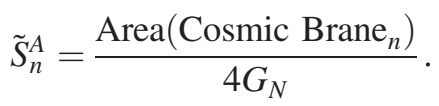

The bulk geometry satisfies the equations of motion (Einstein's equations). In particular, the cosmic brane backreacts on the bulk geometry by creating a conical defect with an opening angle $2 \pi / n$ because of its tension $(n-1) /\left(4 n G_{N}\right)$. Once we know the refined Rényi entropies $\tilde{S}_{n}^{A}$, the Rényi entropy $S_{n}^{A}$ for any positive $n$ is easily obtained by integrating (2): 


$$
S_{n}^{A}=\frac{n}{n-1} \int_{1}^{n} \frac{\tilde{S}_{n^{\prime}}^{A}}{n^{\prime 2}} d n^{\prime}
$$

This holographic prescription for Rényi entropies works in the large $C$ limit of the boundary QFT where $C$ is a generalized "central charge" describing the number of degrees of freedom per short-distance cutoff. In this limit we are instructed to determine a bulk solution with a suitable conical defect. From the bulk perspective, the difference between a canonical and microcanonical ensemble is then expressed as a boundary condition: in the former case we fix the size of the Euclidean time circle on the asymptotic boundary, whereas in the latter we naturally fix the ArnowittDeser-Misner (ADM) energy [14]. We will see that this leads to nontrivial behaviors of the Rényi entropies in a microcanonical ensemble, different from the case of a thermal state.

Finding conical defect solutions with given boundary conditions is a well-defined task, albeit a technically difficult one in general cases without a symmetry. Here, we will simplify this task by focusing on a particular thermodynamic limit with the entire system size $V \rightarrow \infty$ while holding fixed a finite fractional size $f \equiv V_{A} / V$ for $A$ and a finite energy density per "central charge" $\epsilon \equiv E / C V$ (or, equivalently, a finite temperature $T$ in a thermal state). Throughout this Letter, we will refer to this limit simply as the thermodynamic limit with high energy density. In this limit, Rényi entropies are dominated by a volume-law scaling instead of edge effects near the entangling surface $\partial A$, and we will see that from the bulk perspective this volume-law scaling comes from an approximate planar symmetry which allows us to find the relevant conical defect solutions. It is worth noting that the volume-law scaling does not necessarily mean that the Rényi entropy $S_{n}^{A}$ is proportional to the volume $V_{A}$ (in fact we will see that it is not). Rather, it means that $S_{n}^{A} / V_{A}$ approaches a welldefined function of the fractional size $f$ in the $V \rightarrow \infty$ limit. For concreteness, we will first take the holographic $C \rightarrow \infty$ limit and then the $V \rightarrow \infty$ limit.

Our main results in the thermodynamic limit with high energy density are as follows:

1. In a thermal state at inverse temperature $\beta$, the (refined) Rényi entropy of $A$ is simply $f$ times the (refined) Rényi entropy of the entire system:

$$
\begin{aligned}
& \tilde{S}_{n}^{A}(\beta)=f \tilde{S}_{n}(\beta)=f S_{\mathrm{th}}(n \beta), \\
& S_{n}^{A}(\beta)=f S_{n}(\beta)=\frac{f n \beta}{n-1}\left[F_{\mathrm{th}}(n \beta)-F_{\mathrm{th}}(\beta)\right],
\end{aligned}
$$

where $S_{\text {th }}$ is the thermal entropy and $F_{\text {th }}$ is the thermal free energy.

2. In a microcanonical ensemble at fixed energy $E$, the (refined) Rényi entropy of $A$ is

$$
\tilde{S}_{n}^{A}(E)=f S_{\mathrm{th}}\left(n \beta_{n}\right)
$$

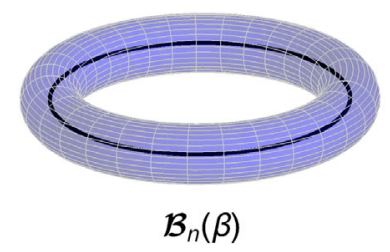

(a)

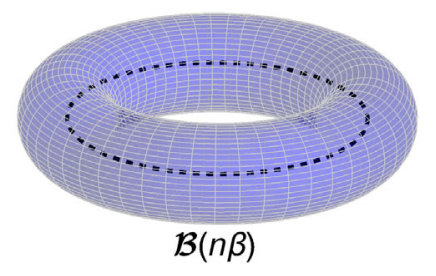

(b)
FIG. 1. (a) Bulk geometry $\mathcal{B}_{n}(\beta)$ with a cosmic brane (black solid circle) homologous to the boundary space $\Sigma$ (a circle here) in a thermal state at inverse temperature $\beta$. (b) Bulk geometry $\mathcal{B}(n \beta)$ with a smooth Euclidean black hole horizon (black dotted circle) in a thermal state at inverse temperature $n \beta$. The left geometry $\mathcal{B}_{n}(\beta)$ can be obtained as a $\mathbb{Z}_{n}$ quotient of the right geometry $\mathcal{B}(n \beta)$, with the cosmic brane identified with the black hole horizon.

$$
S_{n}^{A}(E)=\frac{f S_{\mathrm{th}}\left(n \beta_{n}\right)+(1-f) n S_{\mathrm{th}}\left(\beta_{n}\right)-n S_{\mathrm{th}}\left(\beta_{1}\right)}{1-n},
$$

where $\beta_{n}$ is determined as a function of $n, E$, and $f$ by

$$
E=f E_{\mathrm{th}}\left(n \beta_{n}\right)+(1-f) E_{\mathrm{th}}\left(\beta_{n}\right),
$$

with $E_{\text {th }}$ the expected total energy in a thermal state. The Rényi entropy (8) agrees exactly with the proposal of Ref. [6] for individual energy eigenstates. Here from the holographic perspective, it follows from the much simpler refined Rényi entropy (7) by using Eq. (4).

Thermal state.-In a QFT, a thermal state $e^{-\beta H} / \operatorname{tr}\left(e^{-\beta H}\right)$ on a spatial manifold $\Sigma$ is described by a Euclidean path integral on $\Sigma \times S_{\beta}^{1}$, where $S_{\beta}^{1}$ denotes the Euclidean time circle of size $\beta$. In gauge-gravity duality, the corresponding bulk geometry is a smooth solution to the bulk equations of motion with $\Sigma \times S_{\beta}^{1}$ as the conformal boundary. Let us call this bulk geometry $\mathcal{B}(\beta)$.

In the high temperature limit that we are interested in, the bulk geometry $\mathcal{B}(\beta)$ describes a large Euclidean black hole. This is a solution in which the Euclidean time circle $S_{\beta}^{1}$ contracts to zero size smoothly at the horizon (Fig. 1). The horizon area determines the thermal entropy $S_{\mathrm{th}}(\beta)$ of the entire system [15-20].

Let us first study the Rényi entropies of the entire system. The holographic prescription (3) asks for a bulk solution with a conical defect homologous to the entire $\Sigma$ with a conical opening angle $2 \pi / n$. Let us call this conical geometry $\mathcal{B}_{n}(\beta)$ and the conical defect in it $\mathcal{C}_{n, \beta}$. In fact, $\mathcal{B}_{n}(\beta)$ is easily obtained as a quotient of the smooth geometry $\mathcal{B}(n \beta)$ :

$$
\mathcal{B}_{n}(\beta)=\mathcal{B}(n \beta) / \mathbb{Z}_{n},
$$

where the $\mathbb{Z}_{n}$ group is generated by a shift in the Euclidean time coordinate by $\beta$. The black hole horizon in $\mathcal{B}(n \beta)$ is precisely the set of fixed points under the $\mathbb{Z}_{n}$ action and becomes the conical defect in $\mathcal{B}_{n}(\beta)$ after the $\mathbb{Z}_{n}$ quotient 


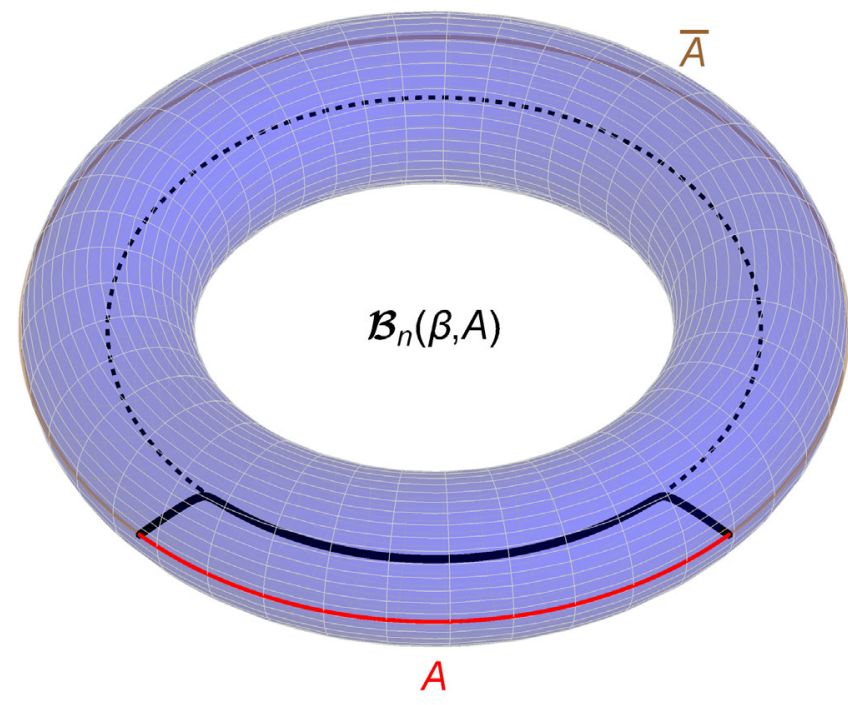

FIG. 2. Bulk geometry $\mathcal{B}_{n}(\beta, A)$ with a cosmic brane (black solid curve) homologous to a subregion $A$ (red interval) in a thermal state at inverse temperature $\beta$. Up to edge effects near $\partial A$, this geometry can be approximately constructed by gluing the segment of the $\mathcal{B}_{n}(\beta)$ geometry corresponding to $A$ and the segment of $\mathcal{B}(\beta)$ corresponding to $\bar{A}$.

(Fig. 1). Therefore, the area of the cosmic brane $\mathcal{C}_{n, \beta}$ in $\mathcal{B}_{n}(\beta)$ is equal to the horizon area in $\mathcal{B}(n \beta)$, and Eq. (3) implies

$$
\tilde{S}_{n}(\beta)=\frac{\operatorname{Area}\left(\mathcal{C}_{n, \beta}\right)}{4 G_{N}}=S_{\mathrm{th}}(n \beta) .
$$

This agrees with the general property that the refined Rényi entropy $\tilde{S}_{n}$ can be defined alternatively as the von Neumann entropy of the density matrix $\rho^{n} / \operatorname{tr} \rho^{n}$.

Now consider the Rényi entropy of a subregion $A$. According to Eq. (3), we need a bulk solution with a conical defect homologous to $A$. Let us call this geometry $\mathcal{B}_{n}(\beta, A)$. It is not easy to construct this geometry exactly, but in the high temperature, thermodynamic limit where the Rényi entropy is dominated by a volume-law scaling instead of edge effects near $\partial A$, the geometry $\mathcal{B}_{n}(\beta, A)$ is approximately given by $\mathcal{B}_{n}(\beta)$ within the bulk subregion $x \in A$ and by $\mathcal{B}(\beta)$ within the subregion $x \in \bar{A}$, where $x$ denotes a collection of coordinates describing directions parallel to $\Sigma$ (Fig. 2). In other words, the geometries $\mathcal{B}_{n}(\beta)$ and $\mathcal{B}(\beta)$ enjoy an approximate planar symmetry in the $x$ directions when we take the high temperature, thermodynamic limit, and the wanted geometry $\mathcal{B}_{n}(\beta, A)$ can be approximated by gluing the part of $\mathcal{B}_{n}(\beta)$ corresponding to $A$ and the part of $\mathcal{B}(\beta)$ corresponding to $\bar{A}$.

According to Eq. (3), the refined Rényi entropy $\tilde{S}_{n}^{A}$ in a thermal state is given by the cosmic brane area in $\mathcal{B}_{n}(\beta, A)$, or $f$ times the cosmic brane area (11) in $\mathcal{B}_{n}(\beta)$ :

$$
\tilde{S}_{n}^{A}(\beta)=f \tilde{S}_{n}(\beta)=f S_{\mathrm{th}}(n \beta) .
$$

Plugging this into Eq. (4), we find the Rényi entropy

$$
S_{n}^{A}(\beta)=f S_{n}(\beta)=\frac{f n \beta}{n-1}\left[F_{\mathrm{th}}(n \beta)-F_{\mathrm{th}}(\beta)\right] .
$$

Even though our results do not require the boundary QFT to have additional symmetries, it is worth considering the special case where it is a $d$-dimensional conformal field theory (CFT), in which the thermal entropy is determined by scale invariance up to a coefficient:

$$
S_{\mathrm{th}}(\beta)=\frac{C V}{\beta^{d-1}} .
$$

Using this, we find that Eqs. (12) and (13) become

$$
\begin{aligned}
& \tilde{S}_{n}^{A}(\beta)=\frac{f C V}{(n \beta)^{d-1}}, \\
& S_{n}^{A}(\beta)=\frac{f C V}{(n \beta)^{d-1}} \frac{n^{d}-1}{d(n-1)} .
\end{aligned}
$$

Microcanonical ensemble.-In a microcanonical ensemble, the state of the entire system is characterized by a fixed total energy $E$ (with some energy width) instead of a fixed Euclidean time circle size $\beta$. Therefore, when applying the holographic prescription (3) for Rényi entropies we need to find a bulk conical solution with the asymptotic boundary condition fixing the ADM energy to be $E$. This prescription can be derived by applying the replica method of Ref. [10] in a microcanonical path integral that fixes the total energy; such microcanonical path integrals were recently studied in Ref. [21] (which was partly motivated by Ref. [22], although there the energy density at each point is fixed instead of just the total energy).

We now show that the required bulk solution is precisely $\mathcal{B}_{n}\left(\beta_{n}, A\right)$ defined earlier with some particular value of $\beta_{n}$ that depends on $n$ as well as implicitly on $E$ and $f$. To see this, we note that the geometry $\mathcal{B}_{n}\left(\beta_{n}, A\right)$ is indeed a bulk solution that has a conical defect homologous to $A$ with the correct conical opening angle $2 \pi / n$. The only remaining condition for $\mathcal{B}_{n}\left(\beta_{n}, A\right)$ to be the correct solution is that its ADM energy should be $E$. In the thermodynamic limit with high energy density, $\mathcal{B}_{n}\left(\beta_{n}, A\right)$ is approximately given by $\mathcal{B}_{n}\left(\beta_{n}\right)$ for $x \in A$ and by $\mathcal{B}\left(\beta_{n}\right)$ for $x \in \bar{A}$ as mentioned earlier. The ADM energy can be calculated by integrating over $\Sigma$ the strength of the gravitational field at the asymptotic boundary. Therefore, the ADM energy of $\mathcal{B}_{n}\left(\beta_{n}, A\right)$ is a weighted average of the ADM energies of $\mathcal{B}_{n}\left(\beta_{n}\right)$ and $\mathcal{B}\left(\beta_{n}\right)$ which we fix to be $E$ :

$$
E=f E_{\mathrm{th}}\left(n \beta_{n}\right)+(1-f) E_{\mathrm{th}}\left(\beta_{n}\right) .
$$

Here we have used that the ADM energy of $\mathcal{B}_{n}\left(\beta_{n}\right)$ is the same as that of $\mathcal{B}\left(n \beta_{n}\right)$ and equal to the expected energy $E_{\mathrm{th}}\left(n \beta_{n}\right)$ in the corresponding thermal state. Equation (17) uniquely determines $\beta_{n}$ as a function of $n, E$, and $f$. 
According to Eq. (3), the refined Rényi entropy $\tilde{S}_{n}^{A}$ in a microcanonical ensemble is determined by the cosmic brane area in $\mathcal{B}_{n}\left(\beta_{n}, A\right)$, which is precisely given by Eq. (12) with $\beta$ set to $\beta_{n}$ :

$$
\tilde{S}_{n}^{A}(E)=f S_{\mathrm{th}}\left(n \beta_{n}\right) .
$$

Again, we determine the Rényi entropy from this by using Eq. (4). The integral with respect to $n$ is nontrivial due to the dependence of $\beta_{n}$ on $n$, but a closed-form expression for the Rényi entropy can be obtained,

$S_{n}^{A}(E)=\frac{f S_{\mathrm{th}}\left(n \beta_{n}\right)+(1-f) n S_{\mathrm{th}}\left(\beta_{n}\right)-n S_{\mathrm{th}}\left(\beta_{1}\right)}{1-n}$,

in terms of $\beta_{n}$ which is defined by Eq. (17).

The microcanonical Rényi entropy (19) is different from the much simpler Rényi entropy (13) in a thermal state, showing that the entanglement spectrum of a subsystem of finite fractional size in a microcanonical ensemble significantly deviates from a purely thermal behavior. In particular, the microcanonical Rényi entropy (19) is not linear in $f$ due to the dependence of $\beta_{n}$ on $f$ : for $n>1(n<1), \beta_{n}$ decreases (increases) with $f$ according to Eq. (17), and Eq. (19) gives a concave (convex) function of $f$ with $\partial^{2} S_{n}^{A}(E) / \partial f^{2}>0(<0)[6]$. On the other hand, the microcanonical Rényi entropy (19) approaches the thermal case (13) at inverse temperature $\beta_{1}$ in two special limits. One is the $f \rightarrow 0$ limit keeping terms that are linear in $f$, in agreement with the derivation of the canonical ensemble for small subsystems from the microcanonical ensemble. The other limit is $n \rightarrow 1$, showing that the von Neumann entropy of a subsystem cannot distinguish microcanonical ensembles from canonical ensembles. This is in contrast to the Rényi entropies which are more sensitive to the finegrained details of a quantum state.

In the special case of a $d$-dimensional CFT, we can use Eq. (14) to find $E_{\mathrm{th}}(\beta)=(d-1) C V /\left(d \beta^{d}\right)$ and solve Eq. (17):

$$
\beta_{n}=\left[\frac{(d-1) C V\left(1-f+f n^{-d}\right)}{d E}\right]^{1 / d} .
$$

Plugging this into Eqs. (18) and (19), we find

$$
\begin{aligned}
& \tilde{S}_{n}^{A}(E)=f S_{\mathrm{mc}}(E) n^{1-d}\left(1-f+f n^{-d}\right)^{(1-d) / d}, \\
& S_{n}^{A}(E)=\frac{n S_{\mathrm{mc}}(E)}{n-1}\left[1-\left(1-f+f n^{-d}\right)^{1 / d}\right],
\end{aligned}
$$

where the microcanonical entropy $S_{\mathrm{mc}}(E)$ is given by

$$
S_{\mathrm{mc}}(E)=C V\left(\frac{d \epsilon}{d-1}\right)^{(d-1) / d},
$$

with $\epsilon \equiv E /(C V)$. The Rényi entropy (22) again agrees exactly with the proposal of Ref. [6] for individual energy eigenstates.

Discussion.-We have shown holographically that Rényi entropies of any subsystem of finite fractional size can be used to diagnose the state of the entire system. In particular, they differ between a microcanonical and a canonical ensemble for the entire system. This is in sharp contrast to the von Neumann entropy which is more coarse-grained and cannot distinguish these two types of ensembles. From the holographic perspective, this is because Rényi entropies are determined from bulk cosmic branes which lead to different backreacted geometries due to distinct boundary conditions in these two types of ensembles, whereas the von Neumann entropy is determined from extremal surfaces that only see the same semiclassical geometry. This distinction between Rényi entropies and the von Neumann entropy is reminiscent of a thermodynamic explanation of a similar phenomenon given in Ref. [23].

The holographic prescription (3) used to calculate the Rényi entropies assumes that the boundary QFT is dual to a bulk theory described by Einstein gravity, but none of our results are modified in more general cases where the bulk theory includes higher-derivative corrections. In these cases, we replace the area of the cosmic brane in Eq. (3) by some generalized notion of area [24-26]. The form of this generalized area is completely determined by the bulk action, but it is sufficient for our purposes to know that the generalized area is an integral over the conical defect of some combination of local geometric invariants. Since our calculation only relies on this locality, the results remain unchanged.

Our results have been obtained in the large $C$ limit of the boundary QFT where the bulk physics is dominated by classical solutions, but again these results are not modified by quantum corrections (at least perturbatively). This is because these quantum corrections take the form of the refined Rényi entropies in the bulk entanglement wedge [26-28], and they scale with the cosmic brane area (and therefore do not affect our calculation) in the thermodynamic limit with high energy density.

For simplicity we have worked in the thermodynamic limit with high energy density so that Rényi entropies satisfy a volume-law scaling in the sense that $S_{n}^{A} / V_{A}$ approaches a well-defined limit. However, our results actually hold in a larger regime defined by only requiring the volume-law term to dominate over edge effects (mainly an area-law term) near the entangling surface $\partial A$. In this larger regime, $S_{n}^{A}$ is well approximated by our results even though it does not necessarily scale with the volume. In the special case of a $d$-dimensional CFT, the larger regime is $E \gg C V^{1-d /(d-1)^{2}} \delta^{-d(d-2) /(d-1)}$ for a microcanonical ensemble or $T \gg V^{-1 /(d-1)^{2}} \delta^{-(d-2) /(d-1)}$ for a canonical ensemble, with $\delta$ the short-distance cutoff (in $d=2$, the powers of $\delta$ are replaced by $\ln \delta$ ). 
Even though we have focused in the previous section on a microcanonical ensemble at fixed energy $E$ (with some energy width), our results (18) and (19) could also apply to an energy eigenstate $|E\rangle$ when the subsystem is no larger than half of the entire system (as proposed in Ref. [6]). Here we provide an intuitive, holographic argument for this. In the case of an energy eigenstate, the cosmic brane prescription (3) for Rényi entropies requires a small modification: instead of requiring the cosmic brane $\mathcal{C}$ to be homologous to the subregion $A$, we only need it to be anchored on the boundary of $A$. Whereas previously the homology constraint requires the existence of a bulk codimension-1 surface $\mathcal{E}$ whose boundary is $A \cup \mathcal{C}$, now we only need the boundary of $\mathcal{E}$ to be $A \cup \mathcal{C}$ up to horizons of pure-state black holes. This modified prescription with a weakened homology constraint is consistent in the sense that it gives the correct, vanishing Rényi entropies for the entire system, as well as the correct von Neumann entropy in two-dimensional holographic CFTs $[29,30]$. It is also reminiscent of the interpretation of the homology constraint given in Refs. [30,31]. Whether this modified prescription can be firmly established for holographic Rényi entropies in general energy eigenstates is an open question that we leave to the future. We would like to mention, however, that two-dimensional CFTs are likely special in this regard, and a different expression for Rényi entropies in Virasoro primary states in the large central charge limit was already obtained in Ref. [32]. This could be explained by the fact that two-dimensional CFTs have an infinite number of commuting conserved charges known as the quantum Korteweg-de Vries (KdV) charges [33], leading to interesting thermodynamic properties [34-41] and differences between primary and descendant states. We leave a full resolution of this puzzle to future work.

I thank Anatoly Dymarsky, Thomas Faulkner, Tarun Grover, Donald Marolf, Henry Maxfield, and Huajia Wang for useful discussions. This work was supported in part by the U.S. Department of Energy under Grant No. DE-SC0019139 and by funds from the University of California. I am also grateful to the KITP for hospitality during part of the development of this work. The KITP was supported in part by the National Science Foundation under Grant No. PHY-1748958.

*xidong@ucsb.edu

[1] J. M. Deutsch, Quantum statistical mechanics in a closed system, Phys. Rev. A 43, 2046 (1991).

[2] M. Srednicki, Chaos and quantum thermalization, Phys. Rev. E 50, 888 (1994).

[3] M. Rigol, V. Dunjko, and M. Olshanii, Thermalization and its mechanism for generic isolated quantum systems, Nature (London) 452, 854 (2008).

[4] A. Rényi, On measures of entropy and information, in Proceedings of the Fourth Berkeley Symposium on
Mathematical Statistics and Probability, Volume 1: Contributions to the Theory of Statistics (University of California Press, Berkeley, California, 1961), pp. 547-561.

[5] R. Islam, R. Ma, P. M. Preiss, M. Eric Tai, A. Lukin, M. Rispoli, and M. Greiner, Measuring entanglement entropy in a quantum many-body system, Nature (London) 528, 77 (2015).

[6] T.-C. Lu and T. Grover, Renyi entropy of chaotic eigenstates, arXiv:1709.08784.

[7] J. R. Garrison and T. Grover, Does a Single Eigenstate Encode the Full Hamiltonian?, Phys. Rev. X 8, 021026 (2018).

[8] A. Dymarsky, N. Lashkari, and H. Liu, Subsystem ETH, Phys. Rev. E 97, 012140 (2018).

[9] A. Lewkowycz and J. Maldacena, Generalized gravitational entropy, J. High Energy Phys. 08 (2013) 090.

[10] X. Dong, The gravity dual of renyi entropy, Nat. Commun. 7, 12472 (2016).

[11] J. M. Maldacena, The Large N limit of superconformal field theories and supergravity, Adv. Theor. Math. Phys. 2, 231 (1998).

[12] S. S. Gubser, I. R. Klebanov, and A. M. Polyakov, Gauge theory correlators from noncritical string theory, Phys. Lett. B 428, 105 (1998).

[13] E. Witten, Anti-de Sitter space and holography, Adv. Theor. Math. Phys. 2, 253 (1998).

[14] R. L. Arnowitt, S. Deser, and C. W. Misner, The dynamics of general relativity, Gen. Relativ. Gravit. 40, 1997 (2008).

[15] J. D. Bekenstein, Black holes and entropy, Phys. Rev. D 7, 2333 (1973).

[16] J. M. Bardeen, B. Carter, and S. W. Hawking, The Four laws of black hole mechanics, Commun. Math. Phys. 31, 161 (1973).

[17] S. W. Hawking, Particle creation by black holes, Commun. Math. Phys. 43, 199 (1975).

[18] G. W. Gibbons and S. W. Hawking, Action integrals and partition functions in quantum gravity, Phys. Rev. D 15, 2752 (1977).

[19] S. Ryu and T. Takayanagi, Holographic Derivation of Entanglement Entropy from AdS/CFT, Phys. Rev. Lett. 96, 181602 (2006).

[20] S. Ryu and T. Takayanagi, Aspects of holographic entanglement entropy, J. High Energy Phys. 08 (2006) 045.

[21] D. Marolf, Microcanonical path integrals and the holography of small black hole interiors, J. High Energy Phys. 09 (2018) 114.

[22] J. David Brown and J. W. York, Jr., The Microcanonical functional integral. 1. The Gravitational field, Phys. Rev. D 47, 1420 (1993).

[23] M. Headrick, General properties of holographic entanglement entropy, J. High Energy Phys. 03 (2014) 085.

[24] X. Dong, Holographic entanglement entropy for general higher derivative gravity, J. High Energy Phys. 01 (2014) 044.

[25] J. Camps, Generalized entropy and higher derivative Gravity, J. High Energy Phys. 03 (2014) 070.

[26] X. Dong and A. Lewkowycz, Entropy, extremality, euclidean variations, and the equations of motion, J. High Energy Phys. 01 (2018) 081. 
[27] T. Faulkner, A. Lewkowycz, and J. Maldacena, Quantum corrections to holographic entanglement entropy, J. High Energy Phys. 11 (2013) 074.

[28] N. Engelhardt and A. C. Wall, Quantum extremal surfaces: Holographic entanglement entropy beyond the classical regime, J. High Energy Phys. 01 (2015) 073.

[29] C. T. Asplund, A. Bernamonti, F. Galli, and T. Hartman, Holographic entanglement entropy from 2d CFT: Heavy states and local quenches, J. High Energy Phys. 02 (2015) 171.

[30] A. Almheiri, X. Dong, and B. Swingle, Linearity of holographic entanglement entropy, J. High Energy Phys. 02 (2017) 074.

[31] D. Harlow, The Ryu-Takayanagi formula from quantum error correction, Commun. Math. Phys. 354, 865 (2017).

[32] T. Faulkner and H. Wang, Probing beyond ETH at large $c$, J. High Energy Phys. 06 (2018) 123.

[33] V. V. Bazhanov, S. L. Lukyanov, and A. B. Zamolodchikov, Integrable structure of conformal field theory, quantum $\mathrm{KdV}$ theory and thermodynamic Bethe ansatz, Commun. Math. Phys. 177, 381 (1996).

[34] J. de Boer and D. Engelhardt, Remarks on thermalization in 2D CFT, Phys. Rev. D 94, 126019 (2016).
[35] P. Basu, D. Das, S. Datta, and S. Pal, Thermality of eigenstates in conformal field theories, Phys. Rev. E 96, 022149 (2017).

[36] S. He, F.-L. Lin, and J.-j. Zhang, Dissimilarities of reduced density matrices and eigenstate thermalization hypothesis, J. High Energy Phys. 12 (2017) 073.

[37] N. Lashkari, A. Dymarsky, and H. Liu, Universality of quantum information in chaotic CFTs, J. High Energy Phys. 03 (2018) 070.

[38] A. Dymarsky and K. Pavlenko, Generalized Gibbs ensemble of $2 \mathrm{~d}$ CFTs at large central charge in the thermodynamic limit, arXiv:1810.11025.

[39] A. Maloney, S. Ng, S. F. Ross, and Ioannis Tsiares, Thermal correlation functions of $\mathrm{KdV}$ charges in 2D CFT, arXiv: 1810.11053.

[40] A. Maloney, S. G. Ng, S. F. Ross, and I. Tsiares, Generalized Gibbs ensemble and the statistics of KdV sharges in 2D CFT, arXiv:1810.11054.

[41] A. Dymarsky and K. Pavlenko, Exact generalized partition function of 2D CFTs at large central charge, arXiv:1812 .05108 